\title{
BOUNDING PATTERNS FOR THE COHOMOLOGY OF VECTOR BUNDLES
}

\author{
MARKUS BRODMANN, ANDRI CATHOMEN, AND BERNHARD KELLER
}

(Communicated by Irena Peeva)

\begin{abstract}
Let $t \in \mathbb{N}$, let $K$ be a field and let $\mathcal{V}_{K}^{t}$ denote the class of all algebraic vector bundles over the projective space $\mathbb{P}_{K}^{t}$.

The cohomology table of a bundle $\mathcal{E} \in \mathcal{V}_{K}^{t}$ is defined as the family of nonnegative integers $h_{\mathcal{E}}:=\left(h^{i}\left(\mathbb{P}_{K}^{t}, \mathcal{E}(n)\right)\right)_{(i, n) \in \mathbb{N}_{0} \times \mathbb{Z}}$.

A set $\mathbb{S} \subseteq\{0, \ldots, t\} \times \mathbb{Z}$ is said to be a bounding pattern for the cohomology of vector bundles over $\mathbb{P}_{K}^{t}$ if for each family $\left(h^{(i, n)}\right)_{(i, n) \in \mathbb{S}}$ of non-negative integers, the set of cohomology tables
\end{abstract}

$$
\left\{h_{\mathcal{E}} \mid \mathcal{E} \in \mathcal{V}_{K}^{t}: h_{\mathcal{E}}^{i}(n) \leq h^{(i, n)} \text { for all }(i, n) \in \mathbb{S}\right\}
$$

is finite. Our main result says that this is the case if and only if $\mathbb{S}$ contains a quasi-diagonal of width $t$, that is, a set of the form

$$
\left\{\left(i, n_{i}\right) \mid i=0, \ldots, t\right\} \text { with integers } n_{0}>n_{1}>\cdots>n_{t} .
$$

\section{INTRODUCTION}

This note continues our investigation [3], which was devoted to the the question "What bounds cohomology of a projective scheme $X$ with coefficients in a coherent sheaf $\mathcal{F}$ ?" The aim of the present paper - which was inspired by the "twin" Master theses [6] and [9] - is to study this question in the special case where $X$ is a projective space over a field and the coherent sheaf of coefficients $\mathcal{F}$ is locally free and hence an algebraic vector bundle.

Our aim is to show that the bounding patterns for the cohomology of vector bundles over projective spaces are the same as the bounding patterns for the cohomology of coherent sheaves over projective varieties. We namely shall prove that a set $\mathbb{S} \subseteq\{0, \ldots, t\} \times \mathbb{Z}$ bounds cohomology (in the sense of [3]) in the class of all pairs $(X, \mathcal{F})$ - where $X$ is a projective scheme over an Artinian ring and $\mathcal{F}$ is a coherent sheaf of $\mathcal{O}_{X}$-modules of dimension $\leq t$ - if and only if it bounds cohomology in the class of all algebraic vector bundles over a projective $t$-space over a given field $K$. To make this precise, we first introduce some notation and recall some basic notions.

Notation 1.1. (A) By $\mathbb{Z}$ we denote the set of integers. If $c \in \mathbb{Z}$ we set $\mathbb{Z}_{\leq c}:=\{n \in$ $\mathbb{Z} \mid n \leq c\}$ and $\mathbb{Z}_{\geq c}:=\{n \in \mathbb{Z} \mid n \geq c\}$. We write $\mathbb{N}:=\mathbb{Z}_{\geq 1}$ and $\mathbb{N}_{0}:=\mathbb{Z}_{\geq 0}$.

(B) Fix $t \in \overline{\mathbb{N}}_{0}$ and let $\mathcal{S}^{t}$ be the class which consists of all pairs $(X, \mathcal{F})$ in which $X=\operatorname{Proj}(R)$ is a projective scheme induced by a Noetherian homogeneous

Received by the editors August 2, 2012 .

2010 Mathematics Subject Classification. Primary 13D45, 13D07; Secondary 14B15.

Key words and phrases. Algebraic vector bundles, boundedness of cohomology, cohomology table. 
ring $R=\bigoplus_{n \geq 0} R_{n}=R_{0}\left[x_{1}, \ldots, x_{r}\right]\left(r \in \mathbb{N}\right.$ and $\left.x_{1}, \ldots, x_{r} \in R_{1}\right)$ over an Artinian base ring $R_{0}$ and $\mathcal{F}$ is a coherent sheaf of $\mathcal{O}_{X}$-modules with $\operatorname{dim}(\mathcal{F}) \leq t$. let

(C) For each pair $(X, \mathcal{F}) \in \mathcal{S}^{t}$, each non-negative integer $i$ and each integer $n$,

$$
h_{\mathcal{F}}^{i}(n)=h^{i}(X, \mathcal{F}(n)):=\text { length }_{R_{0}}\left(H^{i}(X, \mathcal{F}(n))\right.
$$

denote the (finite) $R_{0}$-length of the $i$-th cohomology group $H^{i}(X, \mathcal{F}(n))$ of $X$ with coefficients in the $n$-th twist $\mathcal{F}(n)$ of $\mathcal{F}$.

Definition 1.2. (A) For $(X, \mathcal{F}) \in \mathcal{S}^{t}$ we define the cohomology table of $(X, \mathcal{F})$ as the family of non-negative integers

$$
h_{\mathcal{F}}:=\left(h_{\mathcal{F}}^{i}(n)\right)_{(i, n) \in \mathbb{N}_{0} \times \mathbb{Z}} .
$$

(B) We say that a subset $\mathbb{S} \subseteq\{0, \ldots, t\} \times \mathbb{Z}$ bounds cohomology in the subclass $\mathcal{C} \subseteq \mathcal{S}^{t}$ if for each family $\left(h^{(i, n)}\right)_{(i, n) \in \mathbb{S}}$ of non-negative integers $h^{(i, n)} \in \mathbb{N}_{0}$ the set of cohomology tables

$$
\left\{h_{\mathcal{F}} \mid(X, \mathcal{F}) \in \mathcal{C} \text { and } \forall(i, n) \in \mathbb{S}: h_{\mathcal{F}}^{i}(n) \leq h^{(i, n)}\right\}
$$

is finite. We express this also by saying that the set $\mathbb{S} \subseteq\{0, \ldots, t\} \times \mathbb{Z}$ is a bounding pattern for the cohomology in the class $\mathcal{C}$.

Definition 1.3 (See [3, Definition 4.2]). A subset $\mathbb{T} \subset \mathbb{N}_{0} \times \mathbb{Z}$ is called a quasidiagonal of width $t$ if there is a sequence of integers $\left(n_{i}\right)_{i=0}^{t}$ with $n_{t}<n_{t-1}<\cdots<$ $n_{0}$ such that

$$
\mathbb{T}=\left\{\left(i, n_{i}\right) \mid i=0, \ldots, t\right\} .
$$

The main result of [3] will allow us to say (see Theorem 2.3)

$A$ subset $\mathbb{S} \subseteq\{0, \ldots, t\} \times \mathbb{Z}$ bounds cohomology in $\mathcal{S}^{t}$ if and only if it contains a quasi-diagonal of width $t$.

Clearly, if $\mathbb{S} \subseteq\{0, \ldots, t\} \times \mathbb{Z}$ bounds cohomology in the class $\mathcal{S}^{t}$, it does so in any subclass $\mathcal{C}$ of $\mathcal{S}^{t}$. Conversely, one also might ask for "natural small subclasses" $\mathcal{C}$ of $\mathcal{S}^{t}$ with the property that a set $\mathbb{S} \subseteq\{0, \ldots, t\} \times \mathbb{Z}$ which bounds cohomology in the subclass $\mathcal{C}$ does so in the whole class $\mathcal{S}^{t}$. The aim of this paper is to show that the algebraic vector bundles over a projective space $\mathbb{P}_{K}^{t}$ form such natural small subclasses of $\mathcal{S}^{t}$. To make this precise we introduce the following notation.

Notation 1.4. For a field $K$ we write

$$
\mathcal{V}_{K}^{t}:=\left\{(X, \mathcal{F}) \in \mathcal{S}^{t} \mid X=\mathbb{P}_{K}^{t} \text { and } \mathcal{F} \text { is locally free }\right\}
$$

for the subclass of $\mathcal{S}^{t}$ which consists of all locally free sheaves of finite rank - hence of all algebraic vector bundles - over the projective space $\mathbb{P}_{K}^{t}$.

Now, fix a field $K$. Then, our main result says (see Theorem 4.1)

$A$ subset $\mathbb{S} \subseteq\{0, \ldots, t\} \times \mathbb{Z}$ bounds cohomology in $\mathcal{V}_{K}^{t}$ if and only if it contains a quasi-diagonal of width $t$.

This obviously shows that the set $\mathbb{S} \subseteq\{0, \ldots, t\} \times \mathbb{Z}$ bounds cohomology in the class $\mathcal{S}^{t}$ if and only if it does so in the subclass $\mathcal{V}_{K}^{t}$ of $\mathcal{C}$ (see Corollary 4.3).

It is natural to ask whether in this statement one can replace the class $\mathcal{V}_{K}^{t}$ by its subclass ind $\mathcal{V}_{K}^{t}$ of indecomposable algebraic vector bundles over $\mathbb{P}_{K}^{t}$. In general this is not true (see Example 5.2). But under some additional conditions on the set $\mathbb{S}$ one can indeed replace the class $\mathcal{V}_{K}^{t}$ by its subclass ind $\mathcal{V}_{K}^{t}$ in the above equivalence, as we show in Corollary 5.3. 


\section{BOUNDing PATTERNS FOR THE COHOMOLOGY OF PROJECTIVE SCHEMES}

Throughout this section let $t \in \mathbb{N}_{0}$. We recall a few basic facts which were proved in [3] and we conclude from these that a subset $\mathbb{S} \subseteq\{0, \ldots, t\} \times \mathbb{Z}$ bounds cohomology in the class $\mathcal{S}^{t}$ (see Notation 1.1(B) and Definition 1.2(B)) if and only if it contains a quasi-diagonal of width $t$ (see Definition 1.3).

Reminder 2.1. (A) (See [2, Definition 5.2].) Let $\mathcal{D}^{t+1}$ denote the class of all pairs $(R, M)$ in which $R=\bigoplus_{n \in \mathbb{N}_{0}} R_{n}=R_{0}\left[x_{1}, \ldots, x_{r}\right]\left(r \in \mathbb{N}_{0}\right.$ and $\left.x_{1}, \ldots, x_{r} \in R_{1}\right)$ is a Noetherian homogeneous ring with Artinian base ring $R_{0}$ and $M=\bigoplus_{n \in \mathbb{Z}} M_{n}$ is a finitely generated graded $R$-module with $\operatorname{dim}(M) \leq t+1$.

(B) (See [4, 17.1.4] for example.) If $R=\bigoplus_{n \in \mathbb{N}_{0}} R_{n}$ is a Noetherian homogeneous ring we write $R_{+}$for the irrelevant ideal $\bigoplus_{n \in \mathbb{N}} R_{n}$ of $R$. Moreover by $D_{R_{+}}^{i}(\bullet)$ we denote the $i$-th right derived functor of the $R_{+}$-transform functor $D_{R_{+}}(\bullet)=$ $\underset{n}{\stackrel{\lim }{\longrightarrow}} \operatorname{Hom}_{R}\left(\left(R_{+}\right)^{n}, \bullet\right)$.

Now, let $(R, M) \in \mathcal{D}^{t+1}$. Then, for each $i \in \mathbb{N}_{0}$ the $R$-module $D_{R_{+}}^{i}(M)$ carries a natural grading and for all $n \in \mathbb{Z}$ the $n$-th graded component $D_{R_{+}}^{i}(M)_{n}$ of $D_{R_{+}}^{i}(M)$ is an $R_{0}$-module of finite length. Moreover, $D_{R_{+}}^{i}(M)=0$ for all $i>t$ and $D_{R_{+}}^{i}(M)_{n}=0$ for all $i>0$ and all $n \gg 0$. In particular, for each pair $(i, n) \in \mathbb{N}_{0} \times \mathbb{Z}$ we may define the non-negative integer $d_{M}^{i}(n):=\operatorname{length}_{R_{0}}\left(D_{R_{+}}^{i}(M)_{n}\right)$. This finally allows us to define the cohomology table of $(R, M)$ as the family of non-negative integers

$$
\left(d_{M}^{i}(n)\right)_{(i, n) \in \mathbb{N}_{0} \times \mathbb{Z}} \cdot
$$

(C) We say that a subset $\mathbb{S} \subseteq\{0, \ldots, t\} \times \mathbb{Z}$ bounds cohomology in the subclass $\mathcal{C} \subseteq \mathcal{D}^{t+1}$ if for each family $\left(d^{(i, n)}\right)_{(i, n) \in \mathbb{S}}$ of non-negative integers $d^{(i, n)} \in \mathbb{N}_{0}$ the set of cohomology tables

$$
\left\{d_{M} \mid(R, M) \in \mathcal{C} \text { and } \forall(i, n) \in \mathbb{S}: d_{M}^{i}(n) \leq d^{(i, n)}\right\}
$$

is finite. We express this also by saying that the set $\mathbb{S} \subseteq\{0, \ldots, t\} \times \mathbb{Z}$ is a bounding pattern for cohomology in the class $\mathcal{C}$.

(D) According to [3, Corollary 4.10] a set $\mathbb{S} \subseteq\{0, \ldots, t\} \times \mathbb{Z}$ bounds cohomology in the class $\mathcal{D}^{t+1}$ if and only if $\mathbb{S}$ contains a quasi-diagonal of width $t$ (see Definition 1.3).

Remark 2.2. (A) (See [8, Chapter II] for example.) Keep in mind that there is a surjection

$$
\Phi: \mathcal{D}^{t+1} \rightarrow \mathcal{S}^{t}, \quad(R, M) \mapsto(\operatorname{Proj}(R), \widetilde{M}),
$$

where $\widetilde{M}$ is the sheaf of $\mathcal{O}_{\operatorname{Proj}(R)}$-modules induced by the graded $R$-module $M$.

(B) (See [4, Chapter 20] for example.) For each pair $(R, M) \in \mathcal{D}^{t+1}$ and each pair $(i, n) \in \mathbb{N}_{0} \times \mathbb{Z}$, the Serre-Grothendieck Correspondence yields an isomorphism of $R_{0}$-modules $H^{i}(\operatorname{Proj}(R), \widetilde{M}(n)) \cong D_{R_{+}}^{i}(M)_{n}$, so that $h_{\widetilde{M}}=d_{M}$. Hence, in particular we see that the set $\mathbb{S} \subseteq\{0, \ldots, t\} \times \mathbb{Z}$ bounds cohomology in the class $\mathcal{C} \subseteq \mathcal{D}^{t+1}$ if and only if it bounds cohomology in the class $\Phi(\mathcal{C}) \subseteq \mathcal{S}^{t}$

Theorem 2.3. Let $\mathbb{S} \subseteq\{0, \ldots, t\} \times \mathbb{Z}$. Then $\mathbb{S}$ bounds cohomology in the class $\mathcal{S}^{t}$ if and only if it contains a quasi-diagonal of width $t$.

Proof. This is clear by Reminder 2.1 (D) and Remark 2.2 (B). 
Remark 2.4. (A) If $\mathbb{S} \subseteq\{0, \ldots, t\}$ contains a quasi-diagonal of width $t$, then according to Theorem 2.3 it bounds cohomology in any subclass $\mathcal{C} \subseteq \mathcal{S}^{t}$.

(B) Let $K$ be a field. If the set $\mathbb{S}$ contains a quasi-diagonal of width $t$, then it bounds cohomology in the class $\mathcal{V}_{K}^{t}$ of algebraic vector bundles over $\mathbb{P}_{K}^{t}$.

(C) Observe that all pairs $(X, \mathcal{F}) \in \mathcal{S}^{0}$ have the constant cohomology table $h_{\mathcal{F}}$. So a set $\mathbb{S} \subseteq\{0\} \times \mathbb{Z}$ bounds cohomology in the class $\mathcal{S}^{0}$ if and only if $\mathbb{S} \neq \emptyset$.

\section{Combinatorial Patterns And quasi-Diagonals}

Notation 3.1. (A) Throughout this section, we fix a positive integer $t \in \mathbb{N}$ and a set $\mathbb{S} \subseteq\{0, \ldots, t\} \times \mathbb{Z}$.

(B) We also consider the diagonal projection

$$
\varrho:\{0, \ldots, t\} \times \mathbb{Z} \longrightarrow \mathbb{Z} ; \quad(i, n) \mapsto \varrho(i, n):=i+n .
$$

(C) If $\mathbb{U} \subseteq \mathbb{Z}$ is a set of integers, we form $\inf \mathbb{U}$ and $\sup \mathbb{U}$ within the set $\mathbb{Z} \cup$ $\{-\infty, \infty\}$, with the usual convention that $\inf \emptyset:=\infty$ and $\sup \emptyset:=-\infty$.

Definition 3.2. (A) Let $i \in\{0, \ldots, t\}$. We define the beginning and the end of the set $\mathbb{S}$ at level $i$ respectively by

$$
\begin{aligned}
& \operatorname{beg}^{i}(\mathbb{S}):=\inf \{n \in \mathbb{Z} \mid(i, n) \in \mathbb{S}\} \\
& \operatorname{end}^{i}(\mathbb{S}):=\sup \{n \in \mathbb{Z} \mid(i, n) \in \mathbb{S}\}
\end{aligned}
$$

(B) The height and the depth of $\mathbb{S}$ are defined respectively by

$$
\begin{aligned}
\operatorname{height}(\mathbb{S}) & :=\sup \{i \in \mathbb{Z} \mid \exists n \in \mathbb{Z}:(i, n) \in \mathbb{S}\}, \\
\operatorname{depth}(\mathbb{S}) & :=\inf \{i \in \mathbb{Z} \mid \exists n \in \mathbb{Z}:(i, n) \in \mathbb{S}\} .
\end{aligned}
$$

The width of $\mathbb{S}$ is defined by

$$
w(\mathbb{S}):=\operatorname{height}(\mathbb{S})-\operatorname{depth}(\mathbb{S}) .
$$

Reminder 3.3. (A) According to [1, Definition 1.2], the set $\mathbb{S}$ is called a combinatorial pattern if:

(a) $\operatorname{depth}(\mathbb{S})=0$;

(b) $(i, n) \in \mathbb{S} \Rightarrow \exists j \leq i:(j, n+i-j+1) \in \mathbb{S}$;

(c) $(i, n) \in \mathbb{S} \Rightarrow \exists k \geq i$ : $(k, n+i-k-1) \in \mathbb{S}$;

(d) $0<i \leq t \Rightarrow \operatorname{end}^{i}(\mathbb{S})<\infty$.

(B) The set $\mathbb{S}$ is called a minimal combinatorial pattern if it is a combinatorial pattern and if there is no combinatorial pattern $\mathbb{S}^{\prime} \subsetneq \mathbb{S}$ strictly contained in $\mathbb{S}$.

(C) Let $\mathbb{S}$ be a combinatorial pattern. Then, according to [1, 2.1 and 2.2] the following two statements hold:

(a) The induced projection map $\varrho: \mathbb{S} \rightarrow \mathbb{Z}$ (see Notation 3.1(B)) is surjective.

(b) The map $\varrho: \mathbb{S} \rightarrow \mathbb{Z}$ is bijective if and only if the combinatorial pattern $\mathbb{S}$ is minimal.

(D) Let

$$
\mathbb{F}^{t}:=\left\{\underline{a}:=\left(a_{j}\right)_{j=1}^{p} \mid p \in \mathbb{N}_{0} \text { and } 1 \leq a_{1} \leq a_{2} \leq \cdots \leq a_{p} \leq t-1\right\}
$$

be the set of all monotonically increasing sequences in $\{1, \ldots, t-1\}$. According to the properties of cohomological patterns (a)-(d) of part (A) and statement (b) of 
part $(\mathrm{C})$ there is a bijection between the set $\mathbb{Z} \times \mathbb{F}^{t}$ and the set of all minimal combinatorial patterns of width $t$. Indeed, for each $b \in \mathbb{Z}$ and each $\underline{a}=\left(a_{1}, \ldots, a_{p}\right) \in \mathbb{F}^{t}$ $\left(p \in \mathbb{N}_{0}\right)$, the set

$$
\mathbb{M}_{b, \underline{a}}^{t}:=\left(\{0\} \times \mathbb{Z}_{\geq b}\right) \cup\left\{\left(a_{j}, b-j-a_{j}\right) \mid 1 \leq j \leq p\right\} \cup\left(\{t\} \times \mathbb{Z}_{\leq b-t-p-1}\right)
$$

is a minimal combinatorial pattern of width $t$. Conversely, each minimal combinatorial pattern of width $t$ can be written as $\mathbb{M}_{b, a}^{t}$ with uniquely determined $b \in \mathbb{Z}$ and $\underline{a} \in \mathbb{F}^{t}$.

Our next result has been shown in 9 .

Theorem 3.4. Assume that $\mathbb{S}$ contains no quasi-diagonal of width $t$ and that $\operatorname{beg}^{t}(\mathbb{S}) \neq-\infty$ and $\operatorname{end}^{0}(\mathbb{S}) \neq \infty$. Then, there is a minimal combinatorial pattern $\mathbb{M}$ of width $t$ such that $\mathbb{M} \cap \mathbb{S}=\emptyset$.

Proof. Assume that $\operatorname{end}^{0}(\mathbb{S})=-\infty$, so that $(0, n) \notin \mathbb{S}$ for all $n \in \mathbb{Z}$. Choose an integer $c \leq \operatorname{beg}^{t}(\mathbb{S})$. Then, the minimal combinatorial pattern (see Reminder 3.3)

$$
\mathbb{M}_{c+t, \emptyset}^{t}=\left(\{0\} \times \mathbb{Z}_{\geq c+t}\right) \cup\left(\{t\} \times \mathbb{Z}_{\leq c-1}\right)
$$

is disjoint to $\mathbb{S}$.

Assume now that $\operatorname{end}^{0}(\mathbb{S}) \neq-\infty$, so that $\operatorname{end}^{0}(\mathbb{S}) \in \mathbb{Z}$. Set

$$
b:=\operatorname{end}^{0}(\mathbb{S})+1 \text { and } c:=\operatorname{beg}^{t}(\mathbb{S}) .
$$

First, assume that $t=1$. As $\mathbb{S}$ contains no quasi-diagonal of width $t$, we then have $c \geq b-1$ so that $\mathbb{M}_{b, \emptyset}^{1}=\left(\{0\} \times \mathbb{Z}_{\geq b}\right) \cup\left(\{1\} \times \mathbb{Z}_{\leq b-2}\right)$ is disjoint to $\mathbb{S}$.

So, assume from now on that $t>1$. Our aim is to construct a sequence of integers $\left(a_{1}, a_{2}, \ldots, a_{p}\right)=\underline{a} \in \mathbb{F}^{t}$ with $p \in \mathbb{N}_{0}$ such that $\mathbb{M}_{b, a}^{t} \cap \mathbb{S}=\emptyset$. To achieve this, we construct a sequence of integers $0=: a_{0}<a_{1} \leq a_{2} \leq \cdots \leq a_{i} \leq a_{i+1} \leq \cdots \leq a_{p}<t$ with the following properties:

(1) $\left(a_{i}, b-i-a_{i}\right) \notin \mathbb{S}$ for all $i \in\{0, \ldots, p\}$.

(2) If $0 \leq i<p$ and $a_{i}<a_{i+1}$, then $(j, b-i-j-1) \in \mathbb{S}$ for all $j$ with $a_{i} \leq j<a_{i+1}$.

(3) $p \geq b-c-t$.

By our choice of $b$ and $a_{0}$, condition (1) is satisfied with 0 instead of $p$. If $b-c-t \leq 0$ we can set $p=0$ and the requested sequence is constructed.

Thus, assume from now on that $b-c-t>0$, so that $c<b-t$. As $(0, b-1),(t, c) \in$ $\mathbb{S}$ and $\mathbb{S}$ contains no quasi-diagonal of width $t$, we have

$$
1 \leq a_{1}:=\inf \left\{k \in \mathbb{N}_{0} \mid(k, b-k-1) \notin \mathbb{S}\right\} \leq t-1 .
$$

For this choice of $a_{1}$, condition (2) is satisfied with 1 instead of $p$.

Assume now that the integers $a_{0}<a_{1} \leq \cdots \leq a_{s}<t(s \in \mathbb{N})$ are already constructed such that conditions (1) and (2) are satisfied with $s$ instead of $p$.

If $s \geq b-c-t$, we set $p:=s$ and condition (3) is satisfied. So the requested sequence is constructed in this case. Therefore, it remains to define $a_{s+1}$ if $s<$ $b-c-t$. To do so, we distinguish two cases.

If $\left(a_{s}, b-s-a_{s}-1\right) \notin \mathbb{S}$, we set $a_{s+1}:=a_{s}$. Then, clearly, the sequence $a_{0}, a_{1}, \ldots, a_{s+1}$ satisfies requirements (1) and (2) with $s+1$ instead of $p$ and we have extended our sequence in the requested way. 
So, finally assume that $\left(a_{s}, b-s-a_{s}-1\right) \in \mathbb{S}$. We find integers $0=i_{0}<i_{1}<$ $\cdots<i_{r}<p\left(r \in \mathbb{N}_{0}\right)$ such that

$$
\left\{i_{0}, \ldots, i_{r}\right\}=\left\{i \mid 0 \leq i<s \text { and } a_{i}<a_{i+1}\right\} .
$$

Set

$$
\begin{aligned}
& \mathbb{T}_{1}:=\bigcup_{k=0}^{r}\left\{\left(j, b-i_{k}-j-1\right) \mid a_{i_{k}} \leq j<a_{i_{k}+1}\right\} \text { and } \\
& \mathbb{T}_{2}:=\left\{(j, b-s-j-1) \mid a_{s} \leq j<t\right\} \cup\{(t, c)\} .
\end{aligned}
$$

As $s<b-c-t$ it follows that $\mathbb{T}_{1} \cup \mathbb{T}_{2}$ is a quasi-diagonal of width $t$. Keep in mind that by condition $(1)$ we have $\mathbb{T}_{1} \subseteq \mathbb{S}$. As $\mathbb{S}$ does not contain a quasi-diagonal of width $t$, the set $\mathbb{T}_{2}$ cannot be contained in $\mathbb{S}$. As $\left(a_{s}, b-s-a_{s}-1\right),(t, c) \in \mathbb{T}_{2} \cap \mathbb{S}$ it follows that $(k, b-k-1) \notin \mathbb{S}$ for some $k$ with $a_{s}<k<t$. Choose $k$ minimal with this property and set $a_{s+1}:=k$. Then the sequence $a_{0}, \ldots, a_{s}, a_{s+1}$ satisfies properties (1) and (2) with $s+1$ instead of $p$, and again we have extended our sequence in the requested way.

So altogether, the requested sequence is constructed. Setting $\underline{a}=\left(a_{1}, \ldots, a_{p}\right)$ we easily see by conditions (1) and (3) that indeed $\mathbb{M}_{b, \underline{a}}^{t} \cap \mathbb{S}=\emptyset$.

\section{Bounding PATTERnS For the COHOMOlOgY OF VECTOR BUNDLES}

Throughout this section, let $t \in \mathbb{N}_{0}$. We now are ready to prove the announced main result.

Theorem 4.1. Assume that $\mathbb{S}$ contains no quasi-diagonal of width $t$ and let $K$ be a field. Then, $\mathbb{S}$ does not bound cohomology in the class $\mathcal{V}_{K}^{t}$ of algebraic vector bundles over $\mathbb{P}_{K}^{t}$.

Proof. The case $t=0$ is obvious by Remark 2.4 (C). So, let $t>0$. Assume first, that $\operatorname{beg}^{t}(\mathbb{S})>-\infty$ and $\operatorname{end}^{0}(\mathbb{S})<\infty$. Then, by Theorem 3.4 there is a minimal combinatorial pattern $\mathbb{M}$ of width $t$ such that $\mathbb{M} \cap \mathbb{S}=\emptyset$. According to [1. Proposition 4.5] there is a non-zero locally free indecomposable sheaf $\mathcal{E} \in \mathcal{V}_{K}^{t}$ such that

$$
\mathcal{P}(\mathcal{E}):=\left\{(i, n) \in \mathbb{N}_{0} \times \mathbb{Z} \mid h_{\mathcal{E}}^{i}(n) \neq 0\right\}=\mathbb{M} .
$$

As $\mathcal{E}^{\oplus^{r}} \in \mathcal{V}_{K}^{t}$ and $h_{\mathcal{E} \oplus^{r}}^{i}(n)=r h_{\mathcal{E}}^{i}(n)$ for all $r \in \mathbb{N}$ and all $(i, n) \in \mathbb{N}_{0} \times \mathbb{Z}$, it follows that the set of cohomology tables $\left\{h_{\mathcal{E}^{\oplus}} \mid r \in \mathbb{N}\right\}$ is an infinite subset of

$$
\left\{h_{\mathcal{F}} \mid \mathcal{F} \in \mathcal{V}_{K}^{t}: h_{\mathcal{F}}^{i}(n)=0 \text { for all }(i, n) \in \mathbb{S}\right\} .
$$

Therefore $\mathbb{S}$ does not bound cohomology in the class $\mathcal{V}_{K}^{t}$ in this case.

Assume now that $\operatorname{beg}^{t}(\mathbb{S})=-\infty$ or end ${ }^{0}(\mathbb{S})=\infty$. For each $r \in \mathbb{N}_{0}$, set

$$
\mathbb{S}_{[r]}:=\mathbb{S} \cap(\{0, \ldots, t\} \times\{-r,-r+1, \ldots, r-1, r\}) .
$$

Observe that

$$
\mathbb{S}=\bigcup_{r \in \mathbb{N}_{0}} \mathbb{S}_{[r]}
$$

Clearly, for each $r \in \mathbb{N}_{0}$ the set $\mathbb{S}_{[r]}$ contains no quasi-diagonal of width $t$ and moreover satisfies $\operatorname{beg}^{t}\left(\mathbb{S}_{[r]}\right) \geq-r$ and $\operatorname{end}^{0}\left(\mathbb{S}_{[r]}\right) \leq r$. So, according to Theorem 3.4 , for each $r \in \mathbb{N}_{0}$ there is a minimal combinatorial pattern $\mathbb{M}_{[r]}$ of width $t$ such that

$$
\mathbb{M}_{[r]} \cap \mathbb{S}_{[r]}=\emptyset \text {. }
$$


According to [1, Proposition 4.5], for each $r \in \mathbb{N}_{0}$ we find a locally free sheaf $\mathcal{E}_{[r]} \in \mathcal{V}_{K}^{t}$ such that

$$
\mathcal{P}\left(\mathcal{E}_{[r]}\right):=\left\{(i, n) \in \mathbb{N}_{0} \times \mathbb{Z} \mid h_{\mathcal{E}_{[r]}}^{i}(n) \neq 0\right\}=\mathbb{M}_{[r]} .
$$

Now fix a pair $(i, n) \in \mathbb{S}$. Then there is an integer $s(i, n) \in \mathbb{N}_{0}$ such that $(i, n) \in \mathbb{S}_{[r]}$ for all $r \geq s(i, n)$. Consequently $h_{\mathcal{E}_{[r]}}^{i}(n)=0$ for all $r \geq s(i, n)$. Therefore

$$
h^{(i, n)}:=\sup \left\{h_{\mathcal{E}_{[r]}^{i}}^{i}(n) \mid r \in \mathbb{N}_{0}\right\} \in \mathbb{N}_{0},
$$

and hence

$$
h_{\mathcal{E}_{[r]}}^{i}(n) \leq h^{(i, n)}, \text { for all }(i, n) \in \mathbb{S} \text { and all } r \in \mathbb{N}_{0} .
$$

Our next aim is to show that the set of minimal combinatorial patterns

$$
\mathbb{T}:=\left\{\mathbb{M}_{[r]} \mid r \in \mathbb{N}_{0}\right\}
$$

is not finite. Observe first that for each $r \in \mathbb{N}_{0}$ there is some $n(r) \in \mathbb{N}_{0}$ such that $(t,-n),(0, n) \in \mathbb{M}_{[r]}$ for all $n \geq n(r)$. Assume now that $\mathbb{T}$ is finite, so that there are finitely many integers $r_{1}, \ldots, r_{k}$ with $\mathbb{T}=\left\{\mathbb{M}_{\left[r_{1}\right]}, \ldots, \mathbb{M}_{\left[r_{k}\right]}\right\}$. As $\mathbb{S}=\bigcup_{r \in \mathbb{N}_{0}} \mathbb{S}_{[r]}$ and by our choice of the patterns $\mathbb{M}_{[r]}$, we then obtain

$$
\mathbb{S} \cap \bigcap_{j=1}^{k} \mathbb{M}_{\left[r_{k}\right]}=\emptyset .
$$

It follows that $(t,-n),(0, n) \notin \mathbb{S}$ for all $n \geq m:=\max \left\{n\left(r_{j}\right) \mid j=1, \ldots, k\right\}$. But this implies that $\operatorname{beg}^{0}(\mathbb{S}) \geq-m$ and $\operatorname{end}^{0}(\mathbb{S}) \leq m$, a contradiction!

So, the set $\mathbb{T}$ is infinite, as requested. As a consequence, $\left\{h_{\mathcal{E}_{[r]}} \mid r \in \mathbb{N}_{0}\right\}$ is an infinite subset of

$$
\left\{h_{\mathcal{E}} \mid \mathcal{E} \in \mathcal{V}_{K}^{t}: h_{\mathcal{E}}^{i}(n) \leq h^{(i, n)} \text { for all }(i, n) \in \mathbb{S}\right\} .
$$

This shows that $\mathbb{S}$ does not bound cohomology in $\mathcal{V}_{K}^{t}$.

Corollary 4.2. Let $K$ be a field. Then the set $\mathbb{S} \subseteq\{0, \ldots, t\} \times \mathbb{Z}$ bounds cohomology in the class $\mathcal{V}_{K}^{t}$ of algebraic vector bundles over $\mathbb{P}_{K}^{t}$ if and only if $\mathbb{S}$ contains a quasidiagonal of width $t$.

Proof. This is clear by Theorem 4.1 and by Remark 2.4 (B).

Corollary 4.3. Let $K$ be a field and let $\mathbb{S} \subseteq\{0, \ldots, t\} \times \mathbb{Z}$. Then, the following statements are equivalent:

(i) $\mathbb{S}$ contains a quasi-diagonal of width $t$.

(ii) $\mathbb{S}$ bounds cohomology in the class $\mathcal{S}^{t}$.

(iii) $\mathbb{S}$ bounds cohomology in the class $\mathcal{V}_{K}^{t}$.

Proof. This is clear by Theorem 2.3 , Corollary 4.2 and Theorem 4.1

\section{INDECOMPOSABLE VECTOR BUNDLES}

We keep the hypotheses and notation of the previous sections. It is natural to ask whether one can replace the class $\mathcal{V}_{K}^{t}$ in Corollary 4.2 by the smaller class of all indecomposable vector bundles over $\mathbb{P}_{K}^{t}$. The present section is devoted to this question. We first introduce the following notation. 
Notation 5.1. Let $t \in \mathbb{N}_{0}$ and let $K$ be a field. We write

$$
\text { ind } \mathcal{V}_{K}^{t}:=\left\{\left(\mathbb{P}_{K}^{t}, \mathcal{E}\right) \in \mathcal{V}_{K}^{t} \mid \mathcal{E} \text { is indecomposable }\right\}
$$

for the class of all indecomposable algebraic vector bundles over the projective space $\mathbb{P}_{K}^{t}$.

The following example shows that we cannot expect in general that the class $\mathcal{V}_{K}^{t}$ may be replaced by its subclass ind $\mathcal{V}_{K}^{t}$ in Corollary 4.2

Example 5.2. Let $t=1$ and consider the set $\mathbb{S}:=\{(0,-1),(1,-1)\} \subset\{0,1\} \times \mathbb{Z}$. Clearly, $\mathbb{S}$ does not contain a quasi-diagonal. According to the algebraic form of Grothendieck's Splitting Theorem for vector bundles over the projective line (see [7. Théorème 2.1] or [5, 20.5.9]), (up to isomorphism) the line bundles $\mathcal{O}_{\mathbb{P}_{K}^{1}}(n)$ with $n \in \mathbb{Z}$ are precisely the indecomposable algebraic vector bundles over $\mathbb{P}_{K}^{1}$. So (up to isomorphism) for each choice of $h^{(0,-1)}, h^{(1,-1)} \in \mathbb{N}_{0}$, the $h^{(0,-1)}+h^{(1,-1)}+1$ bundles

$$
\mathcal{O}_{\mathbb{P}_{K}^{1}}(n) \text { with }-h^{(1,-1)} \leq n \leq h^{(0,-1)}
$$

are precisely the indecomposable algebraic vector bundles $\mathcal{E}$ over the projective line $\mathbb{P}_{K}^{1}$ which satisfy $h_{\mathcal{E}}^{i}(n) \leq h^{(i, n)}$ for all $(i, n) \in \mathbb{S}$. Hence

$$
\#\left\{h_{\mathcal{E}} \mid \mathcal{E} \in \text { ind } \mathcal{V}_{K}^{1}: \forall(i, n) \in \mathbb{S}: h_{\mathcal{E}}^{i}(n) \leq h^{(i, n)}\right\} \leq h^{(0,-1)}+h^{(1,-1)}+1 .
$$

This clearly shows that the set $\mathbb{S}$ does indeed bound cohomology in the class ind $\mathcal{V}_{K}^{1}$, although it does not contain a quasi-diagonal.

On the other hand, we have the following result, in which we use the notation

$$
\operatorname{Supp}(h):=\left\{(i, n) \in \mathbb{N}_{0} \times \mathbb{Z} \mid h^{(i, n)} \neq 0\right\}
$$

for a family $h:=\left(h^{(i, n)}\right)_{(i, n) \in \mathbb{N}_{0} \times \mathbb{Z}}$ of non-negative integers $h^{(i, n)}$.

Corollary 5.3. Let $K$ be a field and let $\mathbb{S} \subseteq\{0, \ldots, t\} \times \mathbb{Z}$ be such that one of the following two conditions is satisfied:

(1) $\operatorname{beg}^{t}(\mathbb{S})=-\infty$ or $\operatorname{end}^{0}(\mathbb{S})=\infty$.

(2) There is a finite subset $\mathbb{T} \subset \mathbb{S}$ such that $\mathbb{T} \cap \operatorname{Supp}\left(h_{\mathcal{E}}\right) \neq \emptyset$ for all non-zero bundles $\mathcal{E} \in$ ind $\mathcal{V}_{K}^{t}$

Then the set $\mathbb{S}$ bounds cohomology in the class ind $\mathcal{V}_{K}^{t}$ of indecomposable algebraic vector bundles over $\mathbb{P}_{K}^{t}$ if and only if it contains a quasi-diagonal of width $t$.

Proof. Suppose that $\mathbb{S}$ does not contain a quasi-diagonal of width $t$. We have to show that $\mathbb{S}$ does not bound cohomology in the class ind $\mathcal{V}_{K}^{t}$, provided that one of the two conditions (1) or (2) holds.

Assume first, that condition (1) is satisfied. According to [1, Proposition 4.5] the locally free sheaf $\mathcal{E}_{[r]} \in \mathcal{V}_{K}^{t}$ used in the proof of Theorem 4.1 can be chosen to be indecomposable for all $r \in \mathbb{N}_{0}$. In doing so, we enforce the set $\left\{h_{\mathcal{E}_{[r]}} \mid r \in \mathbb{N}_{0}\right\}$ to be an infinite subset of the set of cohomology tables

$$
\left\{h_{\mathcal{E}} \mid \mathcal{E} \in \text { ind } \mathcal{V}_{K}^{t}: h_{\mathcal{E}}^{i}(n) \leq h^{(i, n)} \text { for all }(i, n) \in \mathbb{S}\right\}
$$

where again $h^{(i, n)}:=\sup \left\{h_{\mathcal{E}_{[r]}}^{i}(n) \mid r \in \mathbb{N}_{0}\right\} \quad\left(\in \mathbb{N}_{0}\right)$ - as in the proof of Theorem 4.1. This shows that $\mathbb{S}$ does not bound cohomology in the subclass ind $\mathcal{V}_{K}^{t}$, and hence proves our claim if condition (1) is satisfied. 
Assume now that condition (2) holds. As $\mathbb{S}$ does not contain a quasi-diagonal of width $t$ it does not bound cohomology in the class $\mathcal{V}_{K}^{t}$, by Corollary 4.2. Consequently there is a family $\left(h^{(i, n)}\right)_{(i, n) \in \mathbb{S}}$ such that the set of cohomology tables

$$
\mathbb{U}:=\left\{h_{\mathcal{E}} \mid \mathcal{E} \in \mathcal{V}_{K}^{t} \text { and } h_{\mathcal{E}}^{i}(n) \leq h^{(i, n)} \text { for all }(i, n) \in \mathbb{S}\right\}
$$

is not finite. Contrary to our claim, we assume now that $\mathbb{S}$ bounds cohomology in the class ind $\mathcal{V}_{K}^{t}$ of indecomposable algebraic vector bundles over $\mathbb{P}_{K}^{r}$. Then, the set

$$
\text { ind } \mathbb{U}:=\left\{h_{\mathcal{E}} \mid \mathcal{E} \in \text { ind } \mathcal{V}_{K}^{t} \text { and } h_{\mathcal{E}}^{i}(n) \leq h^{(i, n)} \text { for all }(i, n) \in \mathbb{S}\right\}
$$

is finite. Moreover, by condition (2), each algebraic vector bundle $\mathcal{E} \in \mathcal{V}_{K}^{t}$ splits in at most

$$
u:=\sum_{(i, n) \in \mathbb{T}} h^{(i, n)} \quad\left(\in \mathbb{N}_{0}\right)
$$

indecomposable summands. So, for each cohomology table $h \in \mathbb{U}$ we may write

$$
h=h_{1}+\ldots+h_{s}, \text { with } 0 \leq s \leq u \text { and } h_{1}, \ldots, h_{s} \in \text { ind } \mathbb{U} .
$$

As ind $\mathbb{U}$ is finite, this yields the contradiction that $\mathbb{U}$ is finite. So, $\mathbb{S}$ does not bound cohomology in the class ind $\mathcal{V}_{K}^{t}$ and this proves our claim.

Remark 5.4. (A) Observe that in Example 5.2 the set $\mathbb{S}$ does not satisfy either of the two conditions (1) or (2) of Corollary 5.3 Observe also that in this example we did use the classification of indecomposable algebraic vector bundles over $\mathbb{P}_{K}^{1}$. A slight modification of the argument performed in Example 5.2 shows indeed that a set $\mathbb{S} \subseteq\{0,1\} \times \mathbb{Z}$ bounds cohomology in the class ind $\mathcal{V}_{K}^{1}$ if and only if $w(\mathbb{S})=1$ (see Definition $3.2(\mathrm{~B})$ ).

(B) We do not know whether there is a purely combinatorial necessary and sufficient condition for the set $\mathbb{S}$ to bound cohomology in the class ind $\mathcal{V}_{K}^{t}$ of indecomposable algebraic vector bundles over $\mathbb{P}_{K}^{t}$ if $t>1$.

\section{REFERENCES}

[1] M. Brodmann and M. Hellus, Cohomological patterns of coherent sheaves over projective schemes, J. Pure Appl. Algebra 172 (2002), no. 2-3, 165-182, DOI 10.1016/S00224049(01)00144-X. MR.1906872(2003f:13017)

[2] Markus Brodmann, Maryam Jahangiri, and Cao Huy Linh, Castelnuovo-Mumford regularity of deficiency modules, J. Algebra 322 (2009), no. 8, 2816-2838, DOI 10.1016/j.jalgebra.2009.06.027. MR2560904 (2011d:13016)

[3] Markus Brodmann, Maryam Jahangiri, and Cao Huy Linh, Boundedness of cohomology, J. Algebra 323 (2010), no. 2, 458-472, DOI 10.1016/j.jalgebra.2009.07.032. MR2564850 (2011a:13035)

[4] M. P. Brodmann and R. Y. Sharp, Local cohomology: an algebraic introduction with geometric applications, Cambridge Studies in Advanced Mathematics, vol. 60, Cambridge University Press, Cambridge, 1998. MR.1613627 (99h:13020)

[5] M. P. Brodmann and R. Y. Sharp, Local cohomology, 2nd ed., Cambridge Studies in Advanced Mathematics, vol. 136, Cambridge University Press, Cambridge, 2013. An algebraic introduction with geometric applications. MR.3014449

[6] A. Cathomen, Zur Diversität der Kohomologietafeln lokal freier Moduln, Master Thesis, University of Zürich (2010). 
[7] A. Grothendieck, Sur la classification des fibrés holomorphes sur la sphère de Riemann (French), Amer. J. Math. 79 (1957), 121-138. MR0087176 (19,315b)

[8] Robin Hartshorne, Algebraic geometry, Springer-Verlag, New York, 1977. Graduate Texts in Mathematics, No. 52. MR0463157 (57 \#3116)

[9] B. Keller, Endlichkeit der Kohomologie lokal freier Moduln, Master Thesis, University of Zürich (2010).

University of Zürich, Institute of MAthematics, Winterthurerstrasse 190, 8057 ZüRICH, SWITZERLAND

E-mail address: brodmann@math.uzh.ch

University of Zürich, Institute of MAthematics, Winterthurerstrasse 190, 8057 ZÜRICH, SWITZERLAND

E-mail address: a.cathomen@gmail.com

University of Zürich, Institute of Mathematics, Winterthurerstrasse 190, 8057 ZüRICH, SWITZERLAND

E-mail address: benikeller@access.uzh.ch 